\section{X-ray diffraction measurements applied to novel materials}

\section{The pitch}

The development of new materials such as nanotubes, graphene, and metalorganic frameworks has put new demands on materials characterization techniques. Applying x-ray diffraction (XRD) and scattering to new materials requires innovative and nonconventional techniques or variations of standard methods.

Traditionally, two different methodologies for investigating a material's atomic arrangement in the solid state are imaging, which provides real space information, and x-ray scattering, which provides reciprocal space information. Imaging techniques such as scanning electron microscopy, and transmission electron microscopy focus on a small area at atomic or near atomic dimensions, while XRD techniques sample a much larger volume of material. Common XRD techniques include structure solution, crystallite size determination, percent crystallinity, residual stress, and orientation (texture analysis).

Many materials are well-ordered single-crystal materials whose structure and diffraction pattern (Figure 1) have been used to characterize the materials.

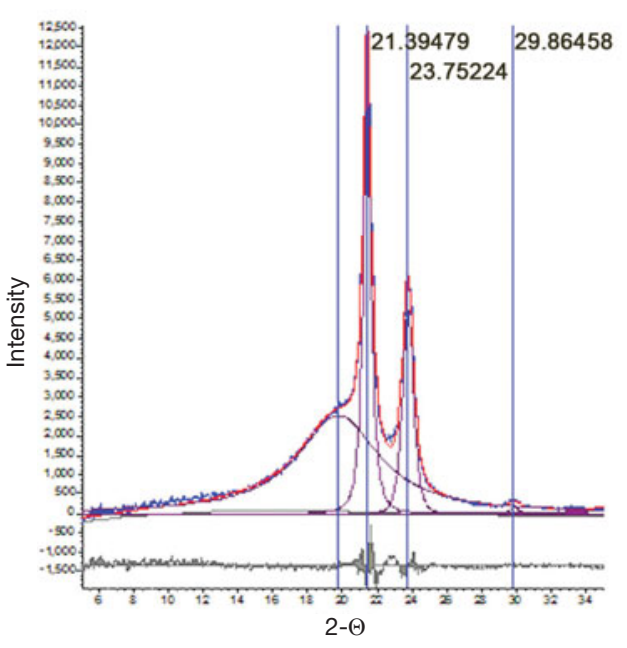

Figure 1. X-ray diffraction pattern showing crystalline and amorphous scattering along with modeled profiles for both components.
However, materials are now being created with only short-range ordering and/or an atomic variation at specific sites in the developed atomic arrangement. While imaging provides insight at or near the sample's surface, XRD techniques offer a probe into the long- and short-range ordering of a large number of scattering moieties beneath the surface. Combining these methodologies provides a more complete understanding of the materials under investigation. X-ray Wizards, a company that specializes in XRD, has worked on solving problems in diverse industries and materials including medical implants, polymers, solar materials, batteries, semiconductors, pharmaceuticals, and ores.

\section{The technology}

X-rays having different wavelengths are used in XRD to expand or reduce the observed range of reciprocal space. Structure elucidation is performed in three ways: single crystal methods, powder diffraction through Rietveld analysis (which uses the molecular structure of the crystallographic phases to determine values for the unit cell, fractional occupancy of atomic sites, and compound quantification) and pair distribution function (PDF) analysis (a method for determining interatomic distances of amorphous, semicrystalline and crystalline materials in real space). For PDF analysis, $0.07 \mathrm{~nm} \mathrm{X}$-rays are used but synchrotron sources can also be used to extend the Q range (defines the range of values used in a particular measurement such as small-angle scattering).

Rietveld techniques for powder samples are used to solve single-crystal structures when crystals have not been suitable in size or quality for traditional single-crystal structure solution methods. The combination of Rietveld and PDF analysis on disordered crystalline material provides both the average structure of the material and atomic displacements at disordered sites.

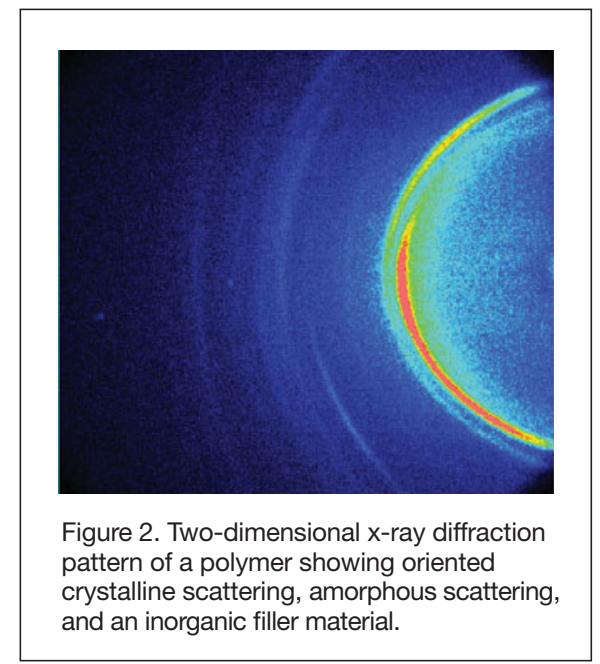

Once a material is structurally characterized, other XRD techniques can be used during the commercialization process. If the material is crystalline, the crystallite size, the degree of crystallinity, or the orientation of the crystallites can be measured and controlled in a fabricated part en route to a final product (Figure 2). While XRD measurements can be made before a material has been structurally characterized, the diffraction results can be put into a clearer context once the structure has been determined.

X-ray Wizards frequently transfers diffraction methods from one field to another. One example is extending the use of micro-XRD (small point source $\mathrm{X}$-ray beams) to measure residual stress or texture on very small areas or curved parts. This includes residual stress measurements on formed parts for medical implants or for small patterned areas on semiconductor wafers.

\section{Opportunities}

$\mathrm{X}$-ray Wizards is seeking partnerships in the development of specialized $\mathrm{x}$-ray diffraction software. These applications are industry-specific and would fill the needs of nonspecialists in X-ray diffraction. In addition, X-ray Wizards has an opportunity for a forward-looking company in a joint development of a real-time twodimensional detector. The detector, which is currently under development, is scalable and is sensitive to wavelengths used in $\mathrm{x}$-ray diffraction.

Source: Richard Ortega, X-ray Wizards, 41617 Swiftwater Drive, Leesburg, VA 20176 USA; tel. 703-574-5445;

email inquiry@x-raywizards.com; and www.x-raywizards.com. 